\title{
ON THE CORRESPONDENCE OF THE EXPERIMENTAL EEL SPECTRA WITH THE ELECTRON STRUCTURE OF TUNGSTEN
}

\author{
J.J. Czyżewiski, J. Krajniak and S. Klein \\ Institute of Experimental Physics, University of Wrocław \\ Cybulskiego 36, 50-205 Wrocław, Poland \\ (Received May 21, 1991)
}

\begin{abstract}
In the previous paper [7] we have directly compared the experimental Electron Energy Loss (EEL) spectra with both the theoretical bulk density-of-states (BDOS) and surface density-of-states (SDOS) functions for tungsten. On the base of the ILEED intensity calculations we have estimated to what extend an EEL spectrum measured at some value of the primary electron energy $E_{\mathrm{p}}$, should correspond either to the SDOS or to the BDOS. Also, the angular acceptance of a CMA has been used to separate the EEL spectra measured as a function of $E_{\mathrm{p}}$ which corresponded either to the [001] direction or to the [011] in parallel to the studied (001) surface. In the present paper we propose a procedure which combines the Electron Energy Loss Spectra (EELS) simulation method based on Bauer's theory [1,2] and the inelastic low energy diffraction (ILEED). If the standard simulation procedure is applied for tungsten an ambiguity would arise because the DOS function is very anisotropic and varies as a function of both the direction along the surface and the depth considered within several atomic layers. The application of ILEED has supplemented the simulation procedure with the method which identifies both the correspondence of a reciprocal-lattice vector to a measured EEL spectrum and the localization of a studied electron structure with regard to the distance from the surface.
\end{abstract}

PACS numbers: $79.20 . \mathrm{Fv}, 61.80 . \mathrm{Mk}, 82.65 .-\mathrm{i}$

\section{Introduction}

In the literature [1-7] the electron energy loss spectra (EELS) have often been explained by inter- or intra-band transitions. In fact, the method supplies information either about the surface-localized electron structure within few layers beyond a crystal surface or about the bulk structure with reference to the primary electron energy $E_{\mathrm{p}}$, i.e. to the fitting of an electron wavelength to the reciprocal-lattice vectors. Taking into account the experimental results presented in our previous paper [7], we have found the procedure of the EELS simulation 
[1-3] to be hardly applicable if the inelastic low energy diffraction (ILEED) effects were not taken into account. We have already shown $[6,7]$ some influence of the ILEED on the results taken with the angular-resolved EEL spectroscopy. The ILEED effects observed in the experiment may help to identify the reciprocal lattice vector $K$ along which the band transitions were investigated and thus to refer the one-dimensional (1D) density-of-states (DOS) function along that $\boldsymbol{K}$ to a measured spectrum. Also, the ILEED phenomenon may identify whether the experimental EELS spectrum carries the information which corresponds to the surface-localized density-of-states function (SDOS) or to the bulk (BDOS).

\section{Theoretical considerations}

The experimental EEL spectra selected and presented in this paper have been shown to be amplified due to diffraction [7]. In that case both the loss energy must be sharply defined and the loss process should be localized around an ion core of the crystal. Then the height of an EELS peak can be considered as the ILEED intensity. That means the inelastic scattering can produce the coherent electron beam whose energy can be analyzed by an angular-resolved EELS taking into account the Bragg condition [8-10]. We presume the analysis selects the electron transitions in tight-binding $d$ bands which occur between the sharp peaks of a DOS function and are as well quasi-localized.

The problem how to refer an experimental EELS spectrum to a theoretical electron structure has been widely discussed in the literature $[1-3,6,7]$. Bauer has found a theoretical expression for the generalized joint density-of-states function. If the DOS function is known an EELS spectrum can be simulated in accordance with the procedure proposed by Kleint et al. which was designed on the base of Bauer's theory [1]. The procedure can be described by

$$
I(\Delta E) \propto \sum_{\mathrm{c}, \mathrm{v}} \int_{\mathrm{v}} \mathrm{d} E_{\mathrm{v}}\left|M_{\mathrm{c}, \mathrm{v}}\left(E_{\mathrm{v}} \rightarrow E_{\mathrm{v}}+\Delta E\right)\right|^{2} N_{\mathrm{c}}\left(E_{\mathrm{v}}+\Delta E\right) N_{\mathrm{v}}\left(E_{\mathrm{v}}\right),
$$

where $N_{\mathrm{c}}$ and $N_{\mathrm{v}}$ are the conduction- and valence-band density-of-states, $M_{\mathrm{c}, \mathrm{v}}$ is the energy-dependent matrix element.

In order to test the procedure we have used the semi-free-electron model in the elementary two-band approximation [12]. In the model the DOS function $N(E)$ is

$$
N(E)=\frac{1}{4 \pi^{2}}\left(\frac{2 m}{h^{2}}\right)\left(q_{\|}^{\max }-q_{\|}^{\min }\right),
$$

where $q_{\|}^{\max }$ and $q_{\|}^{\min }$ are the solutions of the equation,

$$
E=h^{ \pm}\left(q_{\|}\right)
$$

where $h^{ \pm}\left(q_{\|}\right)=\frac{h}{2 m}\left[q_{\|}^{2}+\frac{1}{2}\left(K^{2}-2 q_{\|} \cdot K\right)\right] \pm\left\{\left[\frac{h^{2}}{2 m} \frac{1}{2}\left(K^{2}-2 q_{\|} \cdot K\right)\right]^{2}+\left|U_{K}\right|^{2}\right\}^{\frac{1}{2}}$

Figure 1 illustrates results of the EELS simulation by means of the semi-free-electron DOS function, where the expressions (1-3) were used. The resulting EELS spectrum shows rather small structure that may be expected on the base of a 

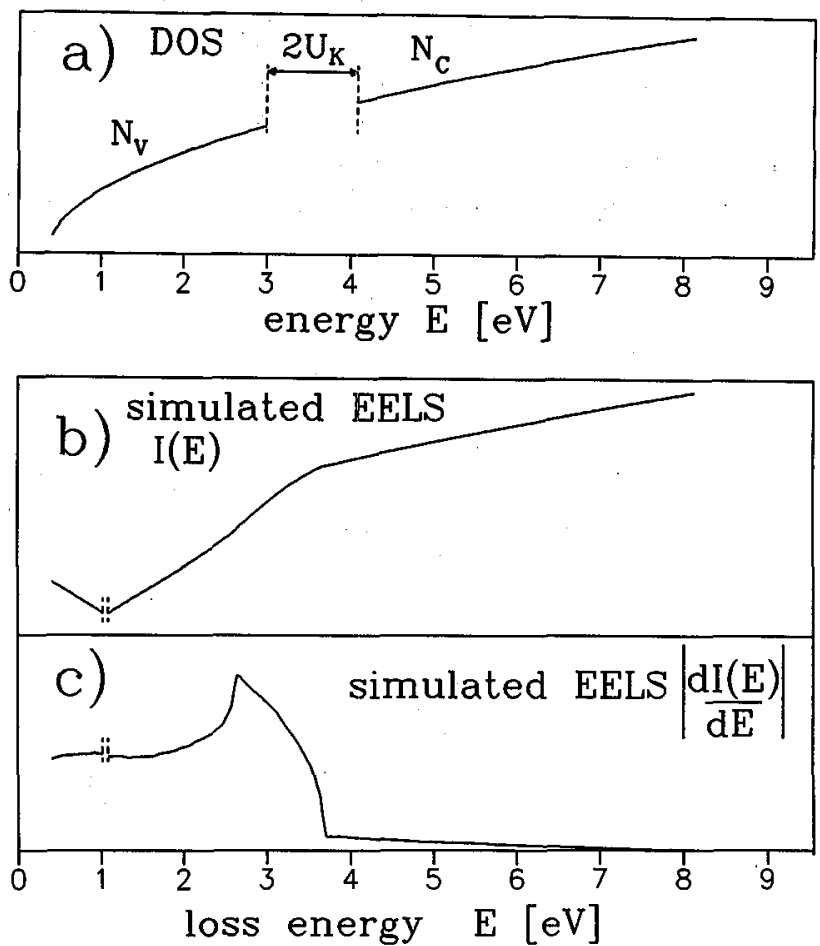

Fig. 1. A simulated EELS spectrum in the semi-free-electron (SFE) model: (a) the SFE density-of-states function, (b) the first derivative EEL spectrum, $I(E)=\mathrm{d} N / \mathrm{d} E$, (c) the second derivative EEL spectrum, $-\mathrm{d}^{2} N / \mathrm{d} E^{2}$.

smooth DOS function. The result may also qualitatively illustrate the negligible contribution of the $s$-band transitions in comparison to the contribution of the $d$-bands in tungsten, because the simulated EELS function presented in Fig. 1b which might approximate the $s$-band EELS is rather smooth.

\section{Results and discussion}

The procedure presented in Fig. 1 has been applied for the investigation of the tungsten electron structure. The experiment has already been described in details in our previous papers $[6,7]$. In short, the (001) face of a tungsten crystal was bombarded by a perpendicular electron beam and the secondary-electron energy was analyzed by a CMA spectrometer. Also, the primary electron energy was varied in steps from $40 \mathrm{eV}$ to $100 \mathrm{eV}$. We have proposed to take into account the angular acceptance of the CMA to observe the ILEED effects in sets of the EEL spectra which were measured as a function of the primary electron energy $E_{\mathrm{p}}$. Taking into account both the CMA angle of acceptance and the surface reverse-lattice vectors of the (001) tungsten plane we could find the $E_{\mathrm{p}}$ regions in which the scattering electrons satisfy the Bragg condition for selected $\boldsymbol{K}$ vectors. 
Three such windows of the $E_{\mathrm{p}}$ values were resolved, i.e. for the [01], [11] and [02] directions along the (001) surface. On the base of our previous results we believe that the EEL spectra measured in each window correspond to the 1D DOS function taken along the reciprocal-lattice vector which refers to the window. Thus, we will discuss here both the theoretical 1D DOS functions and the experimental EEL spectra taken just along the [011] and [001] directions in parallel to the (001) surface.
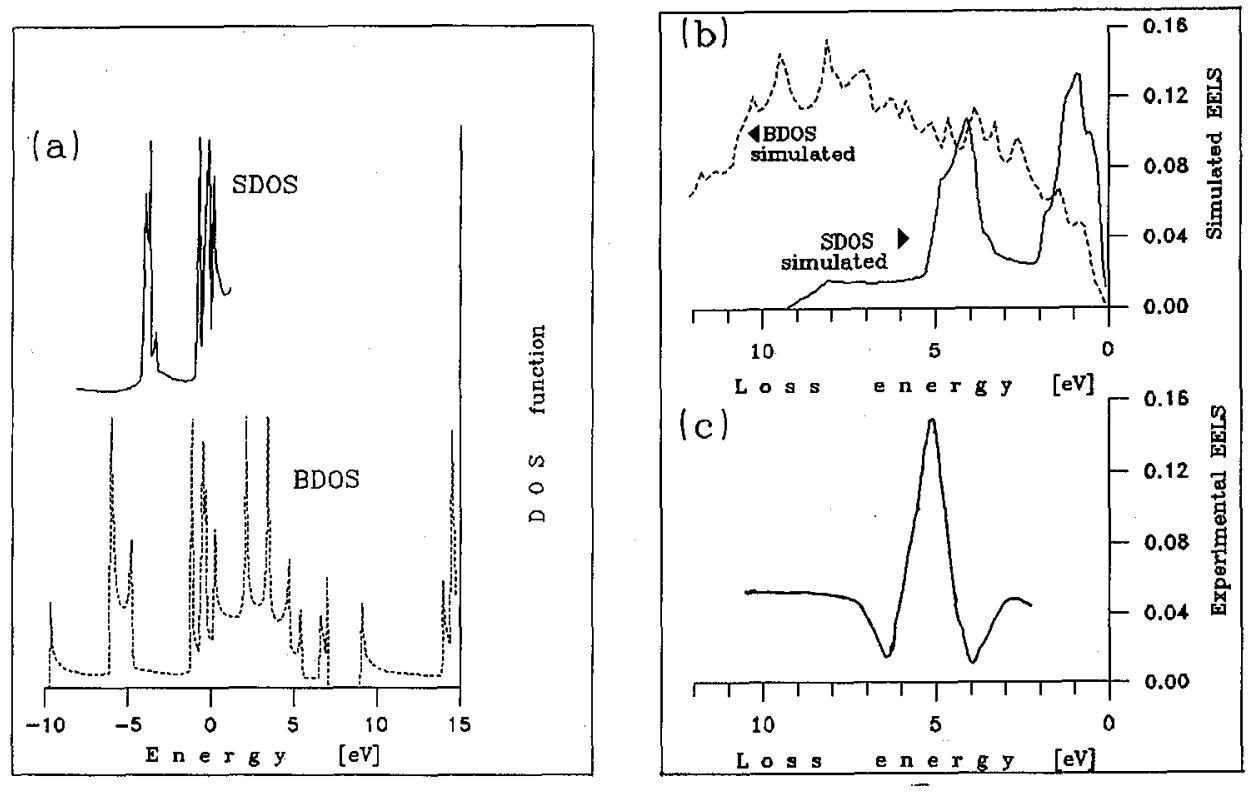

Fig. 2. (a) The surface density-of-states function - SDOS (a solid line) and the bulk BDOS (a dashed line) taken from Ref. [11] for the [001] direction of a tungsten crystal; (b) simulated loss spectra with both the SDOS and the BDOS as presented in (a); (c) the experimental EEL spectrum in the second derivative mode measured at $E_{\mathrm{p}}=100 \mathrm{eV}$ and referred to the [001] direction along the (001) surface of tungsten studied.

In Fig. 2 both the theoretical SDOS and BDOS functions (calculated by Christensen and Feuerbacher [11] - Fig. 2a) and the corresponding simulated EEL spectra (Fig. 2b) as well as the experimental spectrum measured at $E_{\mathrm{p}}=100 \mathrm{eV}$ (Fig. 2c) for the [001] direction of tungsten are collected.

The spectrum measured at $E_{\mathrm{p}}=100 \mathrm{eV}$ is expected to carry the surface message mainly, however, some contribution of bulk electron structure effects may overlap slightly. On the base of the theoretical BDOS [11] for the W[001] plane the overlapping of bulk effects may explain qualitatively the experimental EELS peak at $E=5.0 \mathrm{eV}$ (see Fig. 2c) to be shifted toward a larger value of the loss energy with respect to the corresponding peak in the purely-SDOS-simulated spectrum (see Figs. $2 \mathrm{a}$ and $2 \mathrm{~b}$ : the solid line curves). As a result, we believe the agreement 
between the experimental spectrum and the surface-localized simulated one in the [001] direction is quite good. The bulk simulated spectrum looks entirely different (see Fig. 2b: dashed curve).

In the case of the [011] direction presented in Fig. 3 the situation is quite opposite. Though the analyzer could not resolve all details which we can see in a

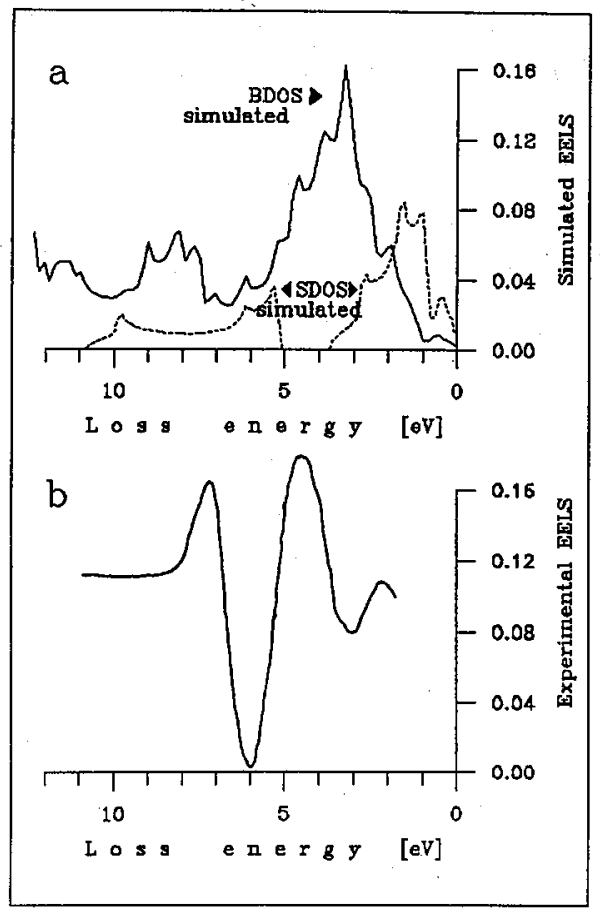

Fig. 3. Simulated loss spectra with both the SDOS (dashed line) and the BDOS (solid line) taken from Ref. [11] for the [011] direction of tungsten (a); the experimental EEL spectrum measured at $E_{\mathrm{p}}=70 \mathrm{eV}$ and referred to the [011] direction along the (001) surface of tungsten studied (b).

bulk simulated spectrum (Figs. $3 \mathrm{a}$ and $3 \mathrm{~b}$ : solid curves), two complexes of peaks, the one at $E=8.5 \mathrm{eV}$ and the second one at about $4 \mathrm{eV}$, correspond to the experimental peaks (Fig. $3 \mathrm{~b}$ ) pretty well. In this case the surface-localized simulated spectrum does not agree with the experimental one measured at $E=70 \mathrm{eV}$. It would support our ILEED calculations [7] which have shown that at $70 \mathrm{eV}$ the inelastic scattered electrons carry the bulk message.

In conclusion one may note that the proposed application of ILEED can supplement the EELS simulation procedure. As a result an improved tool to study both the surface-localized electron structure and the bulk one is obtained. 


\section{References}

[1] E. Bauer, Z. Phys. 224, 19 (1969).

[2] E. Bauer, J. Vac. Sci. Technol. 7, 3 (1969).

[3] Ch. Kleint, M. Funke, R.D. Tomlinson, Appl. Phys. A 46, 137 (1988).

[4] G. Meister, P. Giesert, J. IIölzl, L. Fritsche, Surf. Sci. 143, 547 (1984).

[5] P.E. Luscher, Surf. Sci. 66, 167 (1977).

[6] J.J. Czyżewski, J. Krajniak, Surf. Sci. 231, 18 (1990).

[7] J.J. Czyżewski, J. Krajniak, S. Klein, Surf. Sci. 247, 389 (1991).

[8] J.M. Burkstrand, F.M. Prost, J. Vac. Sci. Technol. 97, 731 (1972).

[9] J.M. Burkstrand, Phys. Rev. B 7, 3443 (1973).

[10] C.B. Duke, U. Landman, Phys. Rev. B 7, 1368 (1973).

[11] N.B. Christensen, E. Feuerbacher, Phys. Rev. B 10, 2349 (1974).

[12] N.W. Ashcroft, N.D. Mermin, Solid State Physics, PWN, Warsaw 1986, p. 212 (Polish edition). 